\title{
Positive Regulation of Oxidative Stress Process
}

National Cancer Institute

\section{Source}

National Cancer Institute. Positive Regulation of Oxidative Stress Process. NCI

Thesaurus. Code C40765.

Oxidative Stress Induction involves initiation of activities that induce a disturbance in the pro-oxidant/antioxidant balance, leading to disruption of cell function by superoxide and other free radicals through damage to DNA bases, protein oxidation products, and lipid peroxidation products. Not synonymous with hypoxia or hyperoxia, oxidative stress promotes a range of degenerative disorders. 